\title{
THE RELATIONSHIPS OF LEPTIN, ADIPONECTIN LEVELS AND PARAOXONASE ACTIVITY WITH METABOLIC AND CARDIOVASCULAR RISK FACTORS IN FEMALES TREATED WITH PSYCHIATRIC DRUGS
}

\author{
Aliye Ozenoglu, ${ }^{\mathrm{I}}$ Huriye Balci, ${ }^{\mathrm{II}}$ Serdal Ugurlu, ${ }^{\mathrm{III}}$ Erkan Caglar, ${ }^{\mathrm{IV}}$ Hafize Uzun, \\ Cihat Sarkis, ${ }^{\text {II }}$ Can Gunay, ${ }^{\text {VI }}$ Engin Eker E ${ }^{\text {I }}$
}

doi: $10.1590 / \mathbf{S 1 8 0 7 - 5 9 3 2 2 0 0 8 0 0 0 5 0 0 0 1 4}$

\begin{abstract}
Ozenoglu A, Balci H, Ugurlu S, Caglar E, Uzun H, Sarkis C et al. The relationships of leptin, adiponectin levels and paraoxonase activity with metabolic and cardiovascular risk factors in females treated with psychiatric drugs. Clinics. 2008; 63(5):651-60.
\end{abstract}

OBJECTIVES: The aim of this study was to investigate serum leptin, adiponectin and paraoxonase1 levels in adult females receiving pharmacotherapy for various psychiatric disorders.

METHODS: The study group consisted of 32 obese females (mean age $40.53 \pm 11.00$ years, mean body mass index $35.44 \pm 5.33$ $\mathrm{kg} / \mathrm{m}^{2}$ ) who were receiving treatment for psychiatric disorders, and the control group included 22 obese females (mean age 35.95 \pm 9.16 years, mean body mass index $30.78 \pm 3.33 \mathrm{~kg} / \mathrm{m}^{2}$ ) who were free of psychiatric disorders. Analyses were performed using a bioelectrical impedance device. Fasting blood samples were obtained for complete blood count and various biochemical tests, including determination of leptin, adiponectin and paraoxonase 1 activity.

RESULTS: Body mass index, waist and hip circumference, body fat percentage, fasting blood glucose, insulin, glycosylated hemoglobin, homeostasis model assesment of insulin resistance, alanine transaminase, aspartate tarnsaminase, and leptin levels were significantly higher in the study group than in controls. Although body weight was positively correlated with leptin levels in both groups, body weight was negatively correlated with adiponectin levels in the control group and positively correlated with adiponectin levels in the study group. In the study group, body mass index and hip circumference correlated positively with leptin levels, hip circumference correlated positively with adiponectin levels, and waist to hip ratio correlated positively with paraoxonase levels. In the control group, body mass index as well as waist and hip circumferences were positively correlated with leptin levels. Weight, body mass index, and hip circumference were also negatively correlated with the adiponectin/leptin ratio in the control group.

CONCLUSION: This study indicates a higher risk for obesity-related disorders, particularly metabolic syndrome, diabetes and cardiovascular disease, in patients treated with psychiatric drugs.

KEYWORDS: Adiponectin; Paraoxonase; Psychiatric drugs; Obesity.

IDepartment of Psychiatry, Cerrahpasa Medical Faculty, University of Istanbul - Istanbul, Turkey.

"Central Laboratory, Cerrahpasa Medical Faculty, University of Istanbul - Istanbul, Turkey.

IIIDepartment of Medicine, Medical Faculty, University of Cumhuriyet Sivas, Turkey.

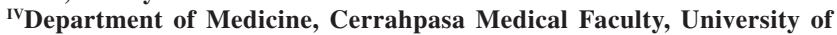
Istanbul - Istanbul, Turkey.

${ }^{v}$ Department of Biochemistry, Cerrahpasa Medical Faculty, University of Istanbul - Istanbul, Turkey.

v'Department of Public Health, Cerrahpasa Medical Faculty, University of Istanbul - Istanbul, Turkey.

Phone: +90 2124143130

Email: aliyeozenoglu@yahoo.com

Received for publication on May 09, 2008

Accepted for publication on July 16, 2008

\section{INTRODUCTION}

Obesity, which is rapidly increasing in prevalence, is closely associated with type 2 diabetes, cardiovascular diseases and hypertension, as well as metabolic syndrome, as well as metabolic syndrome, which is the coexistence of all three of these diseases. In addition to energy storage, adipose tissue also regulates energy metabolism via secreting adipokines into the circulation. Insulin resistance is a primary factor in obesity-related disorders. ${ }^{1}$ By stimulating cellular glucose uptake and acting as an antilipolytic hormone, 
insulin may cause weight gain via direct effects on adipose tissue and influencing appetite through hypoglycemia. Leptin, which is synthesized by adipocytes, regulates appetite and body weight by activating leptin receptors in the satiety center of the hypothalamus. Administration of leptin reduces nutrient intake and body weight. However, it is thought that increased appetite and weight are associated with leptin resistance in the hypothalamus in obese people. It is also known that insulin stimulates leptin synthesis in adipocytes and that, conversely, leptin can both stimulate and inhibit insulin secretion by pancreatic beta cells. Plasma leptin levels have prominent effects on body mass index (BMI) and body fat ratio.

The concentration of adiponectin, a hormone that plays a significant role in regulating insulin sensitivity, is reduced in obesity and type 2 diabetes. ${ }^{2}$ Adiponectin is involved in lipid and glucose metabolism and insulin resistance. ${ }^{3,4}$ Administration of recombinant adiponectin stimulates glucose uptake and lipid oxidation in muscles while reducing lipid uptake and glucose synthesis in the liver, and increases the overall insulin resistance. ${ }^{5}$ In addition to these effects, adiponectin acts as an anti-inflammatory factor and reduces the risk of atherosclerosis, hypertension and coronary heart disease. ${ }^{6,7}$ Excess body fat, particularly abdominal visceral lipid deposition, is frequently accompanied by hypoadiponectinemia, which mediates the relationship between obesity and atherosclerotic vascular diseases. ${ }^{8}$

Hypercholesterolemia, high triglyceride (TG) and low density lipoprotein cholesterol (LDL-C) levels and low high density lipoprotein cholesterol (HDL-C) levels are frequently encountered in obese people. Various studies have shown that obese people have increased oxidative stress as well as greater sensitivity to lipid peroxidation of LDL when compared to healthy controls $(9,10)$. In obesity, LDL oxidation is related to disrupted function of HDL in antioxidant defense; this disruption is mediated by reduced activity of HDL-associated enzymes, such as paraoxanase (HDL-PON1) and lecithincholesterol acyl transferase (11). The risk of diseases related to oxidative damage and lipid peroxidation has been suggested to be greater in people with low paraoxanase1 (PON1) activity than in those with high activity. ${ }^{12}$

Pharmacotherapy of psychiatric diseases results in increased body weight frequently accompanied by increased appetite. This side effect, the occurrence and timing of which is difficult to estimate, eventually leads to obesity, causing cessation of treatment in some patients despite therapeutic success. Obesity also increases the risk of development of various chronic diseases. ${ }^{13-15}$ Weight reduction thus plays a key role in protection from various diseases as well as improved prognoses for diseases that have already developed.
The general clinical approach to psychiatric disorders primarily consists of improving the psychiatric situation. During treatment, patients frequently gain weight and show increased appetite. This increase is generally accepted as the result of recovery and not considered important. However, after pharmacotherapy induced, increased body weight and existing genetic factors could increase the risk of chronic diseases. In this study, several biochemical (leptin, adiponectin and PON1) and anthropometric factors were evaluated with respect to cardiovascular and metabolic disease risks in adult women with psychiatric disorders who became obese after pharmacotherapy.

\section{MATERIALS AND METHODS}

\section{The study population}

The study group included 32 obese adult females (mean BMI $=35.44 \pm 5.33 \mathrm{~kg} / \mathrm{m}^{2}$ ) who were referred to the dietetics department due to complaints about increased body weight while being treated and followed up for psychiatric disorders in the Psychiatry Department of the Cerrahpasa Faculty of Medicine in Istanbul University.

The patients in the study group received pharmacotherapy suitable for their psychiatric conditions (i.e., antidepressants, antipsychotics, mood stabilizers and anxiolytics). Most patients were taking more than one medicine. Their diagnosis had already been performed with suitable psychometric evalutions at the psychiatry clinic and their treatments have been followed by periodic controls. In addition, they had been under psychiatric treatment changing from 1 to 20 years, before referral to our dietetic department. Patients with depression, anxiety, panic, bipolar, obsessive compulsive and psychotic disorders received treatment in our outpatient psychiatry clinic. Patients who were under pharmacotherapy received different medicines and therapies before in long term.

The controls included 22 adult females referred from various clinics to the dietetics department for exogenous obesity (mean BMI $=30.78 \pm 3.33 \mathrm{~kg} / \mathrm{m}^{2}$ ); these patients had no known endocrine or metabolic disorders or history of drug use.

Initially, anthropometric measurements, including height and weight as well as waist and hip circumference were taken. BMI and waist/hip ratios (WHR) were calculated for both groups. Body composition analyses were performed using a bioelectrical impedance analyzer (BIA) device (Bodystat 1500MDD, UK).

\section{Determination of biochemical parameters}

Venous blood samples were drawn into EDTA containing dry tubes without venous stasis after 12 hours overnight 
fasting. Plasma and serum were obtained after at least 30 minutes of clotting by centrifugation at $2500 x \mathrm{x}$ for 15 minutes. Serum were removed and used directly for measurements of biochemical parameters. Other serum and plasma samples were stored at $-70{ }^{\circ} \mathrm{C}$ until assayed for determination of leptin, adiponectin levels and PON1 activity.

Biochemical parameters (serum fasting blood glucose, uric acid, ALT (Alanine transaminase), AST (Aspartate tarnsaminase), triglyceride, total HDL and LDL cholesterol) were measured using an Abbott C8000 automatic analyzer (Abbott, Japan) for all samples. TSH (thyroid stimulating hormone), insulin and cortisol were measured by chemiluminescent immunometric assay using an Immulite 2000 device (DPC; Los Angeles, USA). The plasma homocysteine (Hcy) level was determined by high performance liquid chromatography (HPLC, Agilent 1100 Series). Complete blood count (CBC) was measured using an HMX analyzer (Beckman Coulter, USA). Serum high sensitive $\mathrm{C}$ reactive protein (hsCRP) concentrations were measured using a Behring BN II nephelometer (Dade Behring, Malburg, Germany). Plasma fibrinogen levels were measured using a BCT analyzer (Dade Behring, Malburg, Germany). Serum B12 and folic acid levels were measured by radioimmunoassay (RIA, DPC, USA). Serum ferritins were measured using an Abbott IMX device (Abbott Labs, Abbott Park, IL, USA). HbA1C levels were measured using an HLC-723 G7 HPLC system (Tosoh Corporation, Tokyo, Japan).

Homeostatic model assessment (HOMA) is a method for assessing beta- cell function and insulin resistance (HOMAIR) from basal (fasting) glucose and insulin. HOMA-IR was calculated by the formula [fasting glucose $(\mathrm{mmol} / \mathrm{L}) \mathrm{x}$ fasting insulin $(\mu \mathrm{U} / \mathrm{ml})] / 22.5$. Complete medical history and physical examination were performed for each subjects.

Leptin levels in serum samples were assessed via ELISA using commercial sandwich ELISA kits (DRG Diagnostic, Marburg, Germany).

Serum PON1 activity was assayed using synthetic paraoxon (diethyl-p-nitrophenyl phosphate) as substrate. PONl activity was determined by measuring the initial rate of substrate hydrolysis to p-nitrophenol, which absorbance was monitored at $412 \mathrm{~nm}$ in the assay mixture containing 2.0mM paraoxon, 2, $0 \mathrm{mM} \mathrm{CaCl} 2$ and $20 \mathrm{~L}$ of plasma in 100 $\mathrm{mM}$ Tris-HCI buffer $(\mathrm{pH}=8,0)$. The blank sample containing incubation mixture without plasma was run simultaneously to correct for spontaneous substrate breakdown. The enzyme activity was calculated from E412 of p-nitrophenol (18.290 per $\mathrm{M} / \mathrm{cm}$ ) and was expressed as $\mathrm{U} / \mathrm{ml} ; 1 \mathrm{U}$ of enzyme hydrolyses $1 \mathrm{nmol}$ of paraoxon/min (1). Mean intra-assay and inter-assay coefficients of variation for this analysis were $8.7 \%$ and $9.8 \%$, respectively.
All patient medical and genetic histories, as well as biochemical and anthropometric measurements, were evaluated by internal medicine specialists. Patients with diabetes, dislipidemia, thyroid function disorders or inflammatory and autoimmune disorders were not included in this study. No special tests were used to evaluate the psychiatric conditions of the control group patients. After psychiatric evaluation, patients with disorders requiring treatment were excluded from the study. The nutritional condition of both groups of patients was determined by observing food consumption for the past 7 days and through in-depth interviews with the patients. Variations in weight and appetite of patients during the study were reported from both the records of the outpatient clinics and the patients themselves.

Following measurement and based on information obtained from detailed anamnesis, individualized

individualized nutritional support was initiated for each patient and followed up. However, alterations in the biochemical and anthropometric parameters with the impact of dietary treatment of patients during the follow up period will be described in another study

Statistical analyses were made using Student's $t$, MannWhitney $U$, and Pearson's correlation tests using SPSS for Windows 10.0 software.

\section{RESULTS}

The 32 patients in the study group were using psychiatric drugs for a mean duration of $5.42 \pm 4.91$ years. The drugs included anxiolytics in $2(6.3 \%)$, antidepressants in 27 $(84.4 \%)$, antipsychotics in $10(31.3 \%)$ and mood stabilizers in $11(34.4 \%)$ cases. Most patients have been taken more than one drug.

Patients in the study had been diagnosed with depression (20\%), bipolar disorder (5\%), schizophrenia (3\%), anxiety disorders (2\%), and obsessive compulsive disorder (1\%). Anthropometric measurements of all patients that is in the study group receiving psychiatric pharmacotherapy and control who not receiving any drugs are displayed in Table 1.

For patients in the study group weight, BMI, waist and hip circumference and body fat percentage were significantly higher than those of the controls, while the lean body mass and body water percentage were significantly lower.

Biochemical parameters and leptin, adiponectin and PON1 levels for both groups are compared in Table 2. In the study group blood glucose, insulin, $\mathrm{HbA1c}$, triglyceride, total cholesterol, LDL cholesterol, ferritin, ALT, AST, HOMA-IR and leptin levels were significantly higher than those of the controls, while the adiponectin levels were significantly lower. CRP levels were above reference values $(0-5 \mathrm{mg} / \mathrm{l})$ in 
Table 1 - Anthropometric characteristics of patients receiving and not receiving drugs

\begin{tabular}{|c|c|c|c|}
\hline & $\begin{array}{l}\text { Patients not receiving drugs } \\
\text { Mean } \pm \text { SD }\end{array}$ & $\begin{array}{l}\text { Patients receiving drugs } \\
\text { Mean } \pm \text { SD }\end{array}$ & P-value \\
\hline Age (years) & $35.95 \pm 9.16$ & $40.53 \pm 11.00$ & 0.115 \\
\hline Height $(\mathrm{cm})$ & $155.91 \pm 6.43$ & $156.72 \pm 6.84$ & 0.663 \\
\hline Weight $(\mathrm{kg})$ & $74.86 \pm 9.36$ & $87.25 \pm 15.38$ & 0.001 \\
\hline $\operatorname{BMI}\left(\mathrm{kg} / \mathrm{m}^{2}\right)$ & $30.78 \pm 3.33$ & $35.44 \pm 5.33$ & 0.001 \\
\hline Waist circumference $(\mathrm{cm})$ & $91.19 \pm 7.99$ & $101.56 \pm 10.34$ & 0.000 \\
\hline Hip circumference $(\mathrm{cm})$ & $111.02 \pm 8.05$ & $120.37 \pm 12.39$ & 0.004 \\
\hline Waist/hip ratio & $0.82 \pm 0.04$ & $0.85 \pm 0.05$ & 0.123 \\
\hline Fat mass $(\%)$ & $35.99 \pm 4.44$ & $42.21 \pm 4.77$ & 0.000 \\
\hline Lean mass $(\%)$ & $64.01 \pm 4.44$ & $57.92 \pm 5.18$ & 0.000 \\
\hline Body water (\%) & $46.29 \pm 3.88$ & $42.26 \pm 3.84$ & 0.001 \\
\hline $\operatorname{BMR}(\mathrm{kcal})$ & $1489.90 \pm 119.30$ & $1564.27 \pm 172.25$ & 0.099 \\
\hline
\end{tabular}

BMI: Body mass index, BMR: Basal metabolic rate

Table 2 - Comparison of biochemical findings between groups

\begin{tabular}{|c|c|c|c|}
\hline & $\begin{array}{l}\text { Patients not receiving drugs } \\
\text { Mean } \pm \text { SD }\end{array}$ & $\begin{array}{l}\text { Patients receiving drugs } \\
\text { Mean } \pm \text { SD }\end{array}$ & P-value \\
\hline Glucose (mg/dl) & $86.36 \pm 10.59$ & $95.16 \pm 11.64$ & 0.007 \\
\hline Insulin (micU/ml) & $10.52 \pm 3.68$ & $15.51 \pm 7.52$ & 0.006 \\
\hline $\mathrm{HbA1c}(\%)$ & $5.63 \pm 0.39$ & $5.94 \pm 0.51$ & 0.020 \\
\hline Triglycerides (mg/dl) & $111.86 \pm 62.45$ & $139.94 \pm 59.22$ & 0.029 \\
\hline Total cholesterol (mg/dl) & $182.86 \pm 43.73$ & $203.28 \pm 39.17$ & 0.017 \\
\hline $\mathrm{HDL}(\mathrm{mg} / \mathrm{dl})$ & $43.27 \pm 9.35$ & $47.56 \pm 10.68$ & 0.133 \\
\hline LDL (mg/dl) & $114.35 \pm 30.47$ & $130.44 \pm 27.73$ & 0.049 \\
\hline Hemoglobin (g/dl) & $12.84 \pm 0.81$ & $12.86 \pm 0.84$ & 0.919 \\
\hline Hematocrit (\%) & $38.06 \pm 2.24$ & $37.79 \pm 2.42$ & 0.679 \\
\hline Ferritin (ng/ml) & $21.29 \pm 14.00$ & $33.70 \pm 23.89$ & 0.043 \\
\hline Vitamin $_{12}(\mathrm{pg} / \mathrm{ml})$ & $298.55 \pm 158.32$ & $282.91 \pm 171.85$ & 0.744 \\
\hline Folate (ng/ml) & $4.65 \pm 2.21$ & $5.49 \pm 2.67$ & 0.242 \\
\hline $\operatorname{ALT}(\mathrm{U} / \mathrm{l})$ & $17.17 \pm 5.60$ & $26.17 \pm 14.51$ & 0.016 \\
\hline $\operatorname{AST}(\mathrm{U} / \mathrm{l})$ & $17.50 \pm 4.00$ & $21.21 \pm 6.99$ & 0.047 \\
\hline $\mathrm{CRP}(\mathrm{mg} / \mathrm{l})$ & $4.27 \pm 2.99$ & $7.63 \pm 7.11$ & 0.057 \\
\hline Uric acid (mg/dl) & $4.66 \pm 0.89$ & $4.31 \pm 1.00$ & 0.227 \\
\hline Fibrinogen (mg/dl) & $550.63 \pm 175.33$ & $469.53 \pm 137.11$ & 0.079 \\
\hline Cortisol $(\mu \mathrm{g} / \mathrm{dl})$ & $9.76 \pm 4.31$ & $13.21 \pm 9.20$ & 0.131 \\
\hline Homocysteine $(\mu \mathrm{mol} / \mathrm{l})$ & $10.42 \pm 3.31$ & $10.78 \pm 5.85$ & 0.806 \\
\hline $\mathrm{TSH}(\mathrm{mIU} / \mathrm{ml})$ & $1.91 \pm 0.86$ & $2.28 \pm 3.10$ & 0.588 \\
\hline HOMA-IR & $2.26 \pm 0.88$ & $3.75 \pm 2.12$ & 0.003 \\
\hline Leptin (ng/ml) & $74.49 \pm 25.10$ & $87.75 \pm 18.03$ & 0.028 \\
\hline Adiponectin (ng/ml) & $8.22 \pm 5.07$ & $6.24 \pm 3.73$ & 0.037 \\
\hline PON1 (U/ml) & $258.29 \pm 101.95$ & $232.48 \pm 80.98$ & 0.305 \\
\hline Adiponectin/leptin ratio & $0.15 \pm 0.16$ & $0.08 \pm 0.07$ & 0.073 \\
\hline PON1/HDL ratio & $6.15 \pm 2.45$ & $5.14 \pm 2.39$ & 0.137 \\
\hline
\end{tabular}

HbA1c: glycosilated hemoglobin, TSH: tyroid stimulan hormone, PON1: paraoxanase1, HOMA-IR: homeostasis model assesment of insulin resistance, ALT: Alanine transaminase, AST: Aspartate tarnsaminase 
the study group, while the fibrinogen levels were above the reference range (180-350 mg/dl) in both groups.

Differences in several parameters were re-evaluated following adjustment for age and BMI. When age was adjusted to 38.67 years and BMI was adjusted to $33.54 \mathrm{~kg} /$ $\mathrm{m}^{2}$, the mean adiponectin level was $5.838 \pm 0.709 \mathrm{ng} / \mathrm{ml}$ for patients receiving drugs and $8.804 \pm 0.709 \mathrm{ng} / \mathrm{ml}$ for controls $(\mathrm{p}=0.017)$.

In the controls, leptin levels correlated positively with weight $(\mathrm{r}=0.64 ; \mathrm{p}=0.001)$, BMI $(\mathrm{r}=0.604 ; \mathrm{p}=0.003)$, waist circumference $(r=0.501 ; p=0.021)$, hip circumference $(\mathrm{r}=0.701 ; \mathrm{p}=0.000)$, and Hcy $(\mathrm{r}=0.449 ; \mathrm{p}=0.036)$ and negatively with the adiponectin/leptin ratio $(\mathrm{r}=-0.734$; $\mathrm{p}=0.000$ ).

Also in the controls, adiponectin levels were positively correlated with the PON1 level $(\mathrm{r}=0.433 ; \mathrm{p}=0.044)$, adiponectin/leptin ratio $(\mathrm{r}=0.0753 ; \mathrm{p}=0.000)$, and vitamin $\mathrm{B}_{12}$ level $(\mathrm{r}=0.652 ; \mathrm{p}=0.002)$.

PON1 was only positively correlated with the PON1/ HDL ratio $(\mathrm{r}=0.828 ; \mathrm{p}=0.000)$. The adiponectin/leptin ratio was negatively correlated with weight $(\mathrm{r}=-0.524 ; \mathrm{p}=0.012)$, BMI ( $\mathrm{r}=-0.456 ; \mathrm{p}=0.033)$, hip circumference $(\mathrm{r}=-0.534$; $\mathrm{p}=0.013)$, and Hcy level $(\mathrm{r}=-0.503 ; \mathrm{p}=0.017)$ and positively correlated with the vitamin $B_{12}$ level $(r=0.471 ; p=0.036)$.

Among the study group, leptin levels were positively correlations with weight $(r=0.446 ; p=0.01), B M I(r=0.396$; $\mathrm{p}=0.025)$, and hip circumference $(\mathrm{r}=0.389 ; \mathrm{p}=0.031)$ and negatively correlated with the adiponectin/leptin ratio $(\mathrm{r}=-0.654 ; \mathrm{p}=0.000)$. Adiponectin levels were positively correlated with the adiponectin/leptin ratio $(\mathrm{r}=0.768$; $\mathrm{p}=0.000)$ as well as glucose $(\mathrm{r}=0.365 ; \mathrm{p}=0.04)$, HDL $(\mathrm{r}=0.371 ; \mathrm{p}=0.037)$, and folate levels $(\mathrm{r}=0.430 ; \mathrm{p}=0.014)$ and was negatively correlated with fibrinogen levels $(r=-0.464$; $\mathrm{p}=0.015$ ).PON1 was positively correlated with the PON1/ HDL ratio and WHR $(r=0.743 ; \mathrm{p}=0.000)$. The PON1/HDL ratio was positively correlated with the triglyceride level $(\mathrm{r}=0.377 ; \mathrm{p}=0.033)$ and negatively correlated with the HDL level $(\mathrm{r}=-0.488 ; \mathrm{p}=0.005)$.

\section{DISCUSSION}

During treatment of various diseases, increased body weight is regarded as a sign of improvement. However, druginduced alterations of the mechanisms regulating appetite may result in excessive weight gain and thus endanger compliance with the medical treatment. Various drugs can alter body weight as a negative outcome of their therapeutic effects. ${ }^{16}$ These drugs include psychotropic drugs such as antipsychotics, antidepressants and mood stabilizers. Weight gain is thought to be partially caused by both reduced physical activity caused by the sedative effects of the drugs and excessive consumption of high-calorie drinks.

Excessive fat deposition in adipose tissue and abdominal distribution of this fat negatively influence health in humans. Abdominal obesity is strongly related to disorders involving glucose, insulin and lipid metabolism. ${ }^{17-20}$ In addition to its role in energy storage, adipose tissue also secretes various biologically active adipokines. Among these, leptin and adiponectin have significant roles in insulin sensitivity.

Insulin and leptin play roles in the regulation of body weight. In one study, ${ }^{21}$ the levels of these hormones were investigated in patients treated with various antipsychotics, and patients receiving olanzapine had higher insulin levels than those treated with conventional antipsychotics despite similar BMI values. This situation was attributed to possible effects of olanzapine on insulin secretion. Leptin regulation also changes during olanzapine and clozapine therapy, and the effects of these drugs on insulin and leptin levels were proposed to be associated with the induction of weight gain.

In our study, patients treated with various psychotropic drugs had significantly higher glucose, insulin, HOMA-IR, leptin, triglyceride, total cholesterol and LDL cholesterol levels than the controls despite belonging to the same BMI group (BMI $\left.>30.00 \mathrm{~kg} / \mathrm{m}^{2}\right)$. Body fat mass (\%) as well as waist and hip circumference measurements were also significantly higher in patients receiving drugs. The significantly higher hepatic enzyme levels in the study group may have been due not only to disorders caused by obesity but also to drug therapy. This indicates that psychotropic drugs may increase the risk of developing of metabolic and cardiovascular disorders by affecting hormones related to body weight.

Weight gain caused by psychotropic drug treatment has been proposed to be associated with increased leptin levels. During acute treatment of schizophrenia with clozapine, serum leptin increased two-fold within 2 weeks of initiating therapy and remained high for the first 10 weeks of treatment. Body weight increased by a mean of $4.2 \mathrm{~kg}$ within this period. ${ }^{22}$ Patients treated with clozapine or olanzapine for 4 weeks had mean weight gains of 2.3 and 3.9 $\mathrm{kg}$, respectively; this was compatible with increased leptin secretion. On the other hand, patients who were treated with haloperidol or did not receive psychotropic drugs gained no weight and had stable leptin levels. ${ }^{23}$

Metabolic syndrome is common among patients treated for schizophrenia, ${ }^{24}$ indicating a significant increase in the risk of cardiovascular and metabolic disorders. It has therefore been stressed that an initial evaluation and followup to assess metabolic syndrome-related risks should be a part of the clinical treatment for schizophrenic patients.

In a longitudinal study, ${ }^{25}$ a mean body weight increase of 
$3.3 \mathrm{~kg}$ was associated with a significant increase in plasma leptin levels following the $8^{\text {th }}$ week of acute olanzapine therapy. Within the first 8 weeks of lithium monotherapy, body weights of eight female and seven male inpatients increased by a mean of $5.9 \mathrm{~kg}$, whereas leptin levels increased significantly from a basal value of $6.9 \mathrm{ng} / \mathrm{ml}$ to $10.3 \mathrm{ng} / \mathrm{ml}^{26}$

During 6 weeks of treatment with tricyclic antidepressants and paroxetine, body weight increased by 3.5 and $1.0 \mathrm{~kg}$, respectively, whereas no change was observed in depressed patients who did not receive pharmacotherapy. On the other hand, plasma leptin levels did not change in any of the groups. ${ }^{27}$

In a comparative study in which the effects of atypical antipsychotics, including quetiapine, olanzapine, risperidone and clozapine, on leptin and triglyceride levels and body weight were investigated ${ }^{28}$ olanzapine and clozapine caused significant increases in leptin and triglyceride levels, as well as in body weight. The increases in these parameters were moderate with quetiapine and minimal with risperidone. Also, leptin levels correlated positively with BMI and triglyceride levels.

In our study group, most patients were depressed and receiving antidepressant pharmacotherapy $(62.5 \%)$. The period of treatment and the initial body weights may affect the results of the study. In our study, the leptin level in both groups was positively correlated with body weight, BMI and waist and hip measurements. This result is compatible with previous studies and was expected.

The blood concentrations of adiponectin, which increases insulin sensitivity, are reduced in obesity and type 2 diabetes. ${ }^{2}$ In our study, adiponectin levels were significantly lower in the pharmacotherapy group ( $\mathrm{p}=0.037$, Table 2$)$. In humans, adiponectin levels are known to correlate negatively with body weight, body fat mass and insulin levels.

In addition to its association with lipid and glucose metabolism and insulin resistance, adiponectin also acts

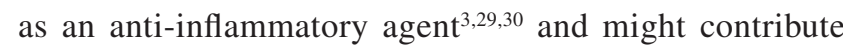
to the development of obesity-related disorders such as atherosclerosis, hypertension and coronary heart disease. ${ }^{6,30,31}$ Excess body fat deposition, particularly that of abdominal visceral fat, is frequently associated with hypoadiponectinemia, a known mediator of the association between obesity and atherosclerotic vascular disorders. ${ }^{8}$

In our study, the mean adiponectin levels of the group using psychotropic drugs were significantly lower than those of the controls (Table 2). Although all the patients in the study were obese $\left(\mathrm{BMI}>30 \mathrm{~kg} / \mathrm{m}^{2}\right)$, there was a significant difference between the BMI values of the two groups. Therefore, several parameters were re-evaluated following adjustment of age and BMI. Following adjustment to an age of 38.67 years and a BMI of $33.54 \mathrm{~kg} / \mathrm{m}^{2}$, mean adiponectin levels were $5.838 \pm 0.709 \mathrm{ng} / \mathrm{ml}$ in the group receiving drugs and $8.804 \pm 0.877 \mathrm{ng} / \mathrm{ml}$ in the control group; this difference was statistically significant $(\mathrm{p}=0.017)$.

Plasma adiponectin and leptin levels, body composition, and glucose consumption were previously investigated in adult females with different ages and obesity levels. ${ }^{32}$ In that study, plasma adiponectin levels did not vary with age but were negatively associated with body fat percentage, visceral fat, subcutaneous abdominal fat, and insulin and leptin levels. Adiponectin levels were also positively correlated with glucose consumption.

In our study, adiponectin levels were positively correlated with age in patients receiving psychiatric drugs $(r=0.525$; $\mathrm{p}=0.002$ ). In patients receiving drugs, adiponectin was positively correlated with glucose, HDL and folate levels as well as the adiponectin/leptin ratio and was negatively correlated with fibrinogen levels. Adiponectin was positively correlated with PON1 and vitamin B $_{12}$ levels as well as the adiponectin/leptin ratio in the control group. These results further suggest the association of adiponectin with cardiovascular diseases.

Hypoadiponectinemia has been reported to be more strongly correlated than other inflammatory indicators with diabetes and metabolic syndrome. ${ }^{33}$ Reduced plasma adiponectin levels are also encountered in conditions that are frequently associated with insulin resistance, such as cardiovascular diseases and hypertension. ${ }^{34,35}$

Both stress and drugs affect the appetite and dietary choices of our patients, who tend to show particularly increased affinity for fatty and sweet foods. The neuroendocrine system is activated by stress, resulting in increased secretion of epinephrine, norepinephrine, cortisol, growth hormone and glucagon. ${ }^{36}$ These neuroendocrine alterations lead to hyperglycemia by affecting the regulation of plasma glucose. In our study, the cortisol levels appeared higher in the group receiving psychiatric drugs, although this difference was not statistically significant (Table 2). Stress-induced activation of the neuroendocrine system and the resulting effects on carbohydrate metabolism may have been responsible for the significantly higher glucose, insulin, HbA1c and HOMA-IR levels in patients who were receiving drugs. The duration of drug therapy was also positively correlated with $\mathrm{HbA1c}$ levels in the group receiving drugs $(r=0.371, p=0.040)$. These results suggest that psychiatric disorders and their corresponding treatments render patients more susceptible to type 2 diabetes mellitus, metabolic syndrome and cardiovascular diseases.

Obesity is an independent risk factor for coronary artery disease and also indirectly increases the risk of coronary artery disease via its associations with insulin resistance, 
hyperlipidemia and hypertension ${ }^{37}$. Visceral adiposity, which describes fat deposition within the abdomen, is highly correlated with an unfavorable coronary risk profile. Therefore, measurements of waist circumference and body fat have become increasingly popular in clinics. A waist circumference of greater than $80 \mathrm{~cm}$ in females indicates increased risk of metabolic syndrome and cardiovascular diseases. ${ }^{38}$ In our study, the waist circumference was greater than this limit in both groups and was also significantly higher in the group receiving psychiatric drugs than in the control group. Hip circumference and body fat percentage were also significance higher in the group receiving drugs (Table 1). While the body fat ratio of controls was in accord with the level expected according to BMI, ${ }^{39}$ it was above the expected value in the group receiving psychiatric drugs.

The presence of metabolic syndrome in patients with type 2 diabetes mellitus indicates a particularly high risk for cardiovascular diseases. ${ }^{40}$ In patients with metabolic syndrome, levels of prothrombotic factors, such as fibrinogen and PAI-1, are usually high. Levels of acute phase reactants such as CRP and fibrinogen as well as those of cytokines (e.g., TNF-alpha and IL-6) are also increased. CRP levels tend to be higher in patients with metabolic syndrome than in healthy individuals. ${ }^{40} \mathrm{~A}$ high CRP level $(>3 \mathrm{mg} / \mathrm{l})$ is considered an overt risk factor for cardiovascular diseases. ${ }^{41}$ In our study, both CRP and fibrinogen levels were high in both groups, while CRP levels were greater than the reference range of our laboratory $(0-5 \mathrm{mg} / \mathrm{l})$ in the group receiving drugs (Table 2 ). In the group receiving psychiatric drugs, adiponectin levels were positively correlated with HDL ( $r=0.371, p=0.037)$ and folate $(r=0.430, p=0.014)$ levels and negatively correlated with fibrinogen levels ( $\mathrm{r}=-0.464$, $\mathrm{p}=0.015$ ); no such correlation of adiponectin with fibrinogen was observed in the controls $(r=-0.100, p=0.667)$. Levels of ferritin, an acute phase reactant, were significantly higher in the patients receiving drugs (Table 2). The parallel increases in levels of inflammatory markers and waist circumference and body fat content in addition to the other biochemical findings support the hypothesis that patients receiving psychiatric drugs are at greater risk for metabolic syndrome and cardiovascular diseases than obese individuals who do not receive psychiatric pharmacotherapy.

In the Amamoto $\mathrm{T}$ at all 's study, the effects of the antipsychotic chlorpromazine on body weight, glucose and lipid metabolism, and adipocytokine secretion were investigated in rats fed with sucrose, ${ }^{42}$ adiponectin levels were significantly higher in the group receiving sucrose than in the controls, whereas they were not altered in the group receiving both sucrose and chlorpromazine. On the other hand, plasma TNF-alpha levels were significantly greater in the group receiving both sucrose and chlorpormazine than in the group receiving sucrose only. Both of these groups had significantly higher fasting glucose and insulin levels than the control group. These results were attributed to inhibition of the adiponectin by chlorpromazine to compensate for increased plasma TNF-alpha levels; this is compatible with obesity.

PON is an enzyme particularly associated with HDL levels that has a significant functional role in the prevention of lipid peroxidation, which increases the tendency of developing atherogenesis and dementia. ${ }^{43,44} 45$

Altered lipid and lipoprotein metabolism in obese individuals have been demonstrated in several studies. Hypercholesterolemia, high triglyceride and LDL levels, and low HDL levels are frequently encountered in obese people. The altered lipoprotein levels and composition are possibly associated with a higher obesity-related risk of cardiovascular diseases. Increased oxidative stress paralleling increased sensitivity of LDL to lipid peroxidation has also been shown in obese individuals. ${ }^{9,10}$ Increased oxidative damage has been probably related to reduced antioxidant capacity. In animal studies, LDL oxidation in obesity was associated with disruption of antioxidant defense by HDL, including reduced activities of HDL-associated enzymes such as paraoxanase (HDL-PON) and lecithin cholesterol acyltransferase. ${ }^{11}$

HDL-PON is a calcium-dependent esterase that can hydrolyze oxidized phospholipids, thereby protecting lipoproteins (LDL, HDL) and membranes from oxidative changes. ${ }^{12}$ The enzymatic activity of PON-HDL varies greatly among healthy people, and individuals with low PON activity are at greater risk for developing diseases involving oxidative stress and lipid peroxidation..$^{12}$ Previous studies have also shown that the antioxidant capacity of HDL and its sensitivity to atherogenic modifications such as oxidation, glycation and homocysteinization are associated with HDLPON activity. ${ }^{46,47}$

In a study that investigated the relationship between HDL-PON activity and oxidative stress of lipoproteins in healthy and obese individuals ${ }^{43}$, HDL-PON activity was significantly lower in obese people than in controls. The negative correlation between HDL-PON activity and lipid peroxide levels suggested an association between paraoxonase activity and lipid peroxidation of lipoproteins. The finding that plasma leptin levels correlated negatively with HDL-PON activity and positively with lipid hydroxy peroxide levels in obese individuals was attributed to the relationship between leptin and oxidative damage of lipoproteins. The authors thus concluded that reduced paraoxonase activity and compositional alterations of HDL and LDL contribute to increased risk of obesity-related cardiovascular diseases. 
The PON/HDL ratio positively correlated with the HOMA index, BMI and $\mathrm{WHR}^{48}$. The authors thus concluded that in a population without hyperglycemia, insulin resistance and hyperinsulinemia are factors contributing to the variability in the intragenotype of paraoxanase activity.

Although the PON1 levels and PON1/HDL ratios appeared in our study in patients receiving drugs lower than controls, the difference was not statistically significant (Table 2). In the patients receiving drugs, PON1 was positively correlated with $\mathrm{BMI}$, while the PON1/HDL ratio was positively correlated with triglyceride levels and negatively correlated with HDL levels. No such association was observed in controls. The results in patients receiving drugs are compatible with other findings indicating increased cardiovascular disease risk.

While previous studies have investigated leptin and adiponectin levels in patients receiving pharmacotherapy for psychiatric disorders, our study is the first in which HDL-PON1 activity was also assessed in addition to a wide spectrum of biochemical parameters.

The epidemiology of obesity plays a significant role in the high prevalence of metabolic syndrome. Abdominal obesity is strongly related to disorders involving glucose, insulin and lipid metabolism. ${ }^{17-20,49}$ Increased nutrient intake, together with inactivity, contributes to increased deposition visceral fat tissue and eventually leads to insulin resistance. The visceral fat mass correlates positively with the severity of insulin resistance..$^{50}$

Depending on the dietary history and food records of patients in study group, it was revealed that while total caloric intake, saturated fat and refined carbohydrate content in their diet were increasing complex carbohydrate, antioxidant vitamins, essential fat and fiber rich foods consumption decreased.

In addition to underlying diseases and pharmacotherapy, lack of essential ingredients in the diet may increase susceptibility to certain chronic diseases. The patients who require pharmacotherapy for psychiatric disorders should be treated and followed up with a multidisciplinary team approach. The presence of a dietician in the treatment team with experience in the areas of endocrinology, metabolism and psychiatry who is also talent in communication skills with patients could be important for treatment success At psychiatric clinics, the development of hormonal disorders and metabolic nutritional alterations are sometimes neglected or not considered important. However, this approach may contribute to the development of other chronic diseases. We therefore believe and recommend that inclusion of a qualified and skilled dietitian could be beneficial when creating the treatment team.

\section{REFERENCES}

1. Yamashita S, Nakamura T, Shimomura I, Nishida M, Yoshida S, Kotani $\mathrm{K}$, et al. Insulin resistance and body fat distribution. Diabetes Care. 1996;19:287-91.

2. Gale SM, Castracane VD, Mantzoros CS. Energy homeostasis, obesity and eating disorders: Recent advances in endocrinology. J Nutr. 2004:134:295-8.

3. Yamauchi T, Kamon J, Waki H, Terauchi Y, Kubota N, Hara K, et al. The fat derived hormon adiponectin reverses insulin resistance associated with both lipoatrophy and obesity. Nat Med. 2001; 7:941-6.

4. Yamauchi T, Kamon J, Minokoshi Y, Ito Y, Waki H, Uchida S. Et al Adiponectin stimulates glucose utilization and fatty acid oxidation by activating AMP-activated protein kinase. Nat Med. 2002;8:1288-95.

5. Heilbronn LK, Smith SR, Ravussin E. The insulin sensitizing role of the fat derived hormone adiponectin. Curr Pharm Design. 2003;9:1411-18.

6. Pischon T, Girman CJ, Hotamisligil GS, Rifai N, Hu FB, Rimm EB Plasma adiponectin levels and risk of myocardial infarction in man. JAMA. 2004;29:1730-7.
7. Spiegelman BM, Choy L, Hotamisligil GS, Graves RA, Tontonoz P. Regulation of adipocyte gene expression in differantiation and syndromes of obesity/diabetes. J Biol Chem. 1993;268:6823-6.

8. Ouchi N, Kihara S, Funahasti T, Matsuzawa Y, Walsh K. Obesity, adiponectin and vascular inflammatory disease. Curr Opin Lipidol. 2003;14:561-6.

9. Mutlu-Turkoglu U, Oztezcan S, Orhan Y, Aykac-Toker G, Sivas A, Uysal $\mathrm{M}$. An increase in lipoprotein oxidation and endogenous lipid peroxides in serum of obese women. Clin Exp Med. 2003;2:171-74.

10. Myara I, Alamowitch C, Michel O, Heudes D, Bariety C, Guy-Grand B, Chevalier J. Lipoprotein oxidation and plasma vitamin $\mathrm{E}$ in nondiabetic normotensive obese patients. Obes Res 2003;11:112-20.

11. Mertens A, Verhamme P, Bielicki JK, Philips MC, Quarck R, Verreth W, Stengel D, Ninio E, Navab M, Macness B, et al. Increased low-density lipoprotein oxidation and impaired high density antioxidant defence are associated with increased macrophage homing and atherosclerosis in dyslipidemic obese mice. LCAT gene transfer decreases atherosclerosis. Circulation. 2003;107; 1640-46. 
12. Durrington PN, Mackness B, Mackness MI. Paraoxanase and aterosclerosis. Arterioscler Tromb Vasc Biol. 2001;2:473-80.

13. Aronne LJ. Epidemiology, morbidity and treatment of overweight and obesity. Journal of Clinical Psychiatry. 2001;62:13-22.

14. Rosmond R, Björntorp P. The role of antidepressants in the treatment of abdominal obesity. Medical Hypotheses. 2000;54:990-94.

15. Özenoğlu A. Medical nutrition therapy in obesity 2001; $1^{\text {st }}$ Edition, İstanbul Dilek Ofset

16. Özenoğlu A. Approaches to the alteration in appetite and body weight during psychiatric diseases treatment. Science of Diabetes. : 4 (1) 34 50

17. Rosmond R, Björntorp P. Endocrine and metabolic aberrations in men with abdominal obesity in relation to anxio-depressive infirmity. Metabolism. 1998;47:1187-93

18. Rosmond R, Dalman MF, Björntorp P. Stress-related cortisol secretion in men: relationships with abdominal obesitl and endocrine, metabolic and hemodynamic abnormalities. J Clin Endocrinol Metab. 1998;83:1853-59.

19. Björntorp P, Holm G, Rosmond R. Hypothalamic arousal, insulin resistance and Type 2 diabetes mellitus. Diabet Med. 1999;16:373-83.

20. Rosmond R, Björntorp P. Blood pressure in relation to obesity, insulin and the hypothalamic-pituitary-adrenal axis in Swedish men. J Hypertens. 1998;16:1721-26.

21. Melkersson KI, Hulting AI. Insulin and leptin levels in patients with schizophrenia or related psychoses-a comparision between different antipsychotic agents. Psychopharmacology. 2001;154: 205-12.

22. Brömel T, Blum WF, Ziegler A, Schulz E, Bender M, Fleischhaker C, et al. Serum leptin levels increase rapidly after initiation of clozapine therapy. Mol Psychiatry. 1998;3:76-80.

23. Kraus T, Haack M, Schuld A, Hinze-Selch D, Kühn M, Uhr M, et al. Body weight and leptin plasma levels during treatment with antipsychotic drugs. Am J Psychiatry. 1999;156:312-4.

24. Hert MA, Winkel RV, Eyck DV, Hanssens L, Wampers M, Scheen A, Peuskens J. Prevalance of the metabolic syndrome in patients with schizophrenia treated with antipsychotic medication. Schizophrenia Research. 2006;83:87-93.

25. Eder U, Mangweth B, Ebenbichler C, Weiss E, Hofer A, Hummer M, et al.Association of olanzapine-induced weight gain with an increase in body fat. Am J Psychiatry. 2001;158:1719-22.

26. Atmaca M, Kulo lu M, Tezcan E, Ustünda B. Weight gain and serum leptin levels in patients on lithium treatment. Neuropsychobiology. 2002;46:67-9.

27. Hinze-Selch D, Deuschle M, Weber B, Heuser I, Pollmächer T. Effect of coadministration of clozapine and fluvoxamine versus clozapine monotherapy on blood cell counts, plasma levels of cytokines and body weight. Psychopharmacology (Berl). 2000;149:163-9.

28. Atmaca M, Kuloglu M, Tezcan E, Ustunda B. Serum leptin and triglyceride levels in patients on treatment with atypical antipsychotics. J Clin Psychiatry. 2003;64:598-604.

29. Kondo H, Shimomura I, Matsukawa Y, Kumada M, Takahashi M, Matsuda M, et al. Association of adiponectin mutation with type 2 diabetes: a candidate gene for the insulin resistance syndrome. Diabetes. 2002; 5:2325-8.
30. Im JA, Kim SH, Lee JW, Shim JY, Lee HR, Lee DC. Association between hypoadiponectinemia and cardiovascular risk factors in nonobese healthy adults. Metabolism Clinical and Experimental 2006;55:154650 .

31. Kumada M, Kihara S, Sumitsuji S, Kawamoto T, Matsumoto S, Ouchi $\mathrm{N}$, et al. Coronary artery disease. Association of hypoadiponectinemia with coronary artery disease in man. Arterioscler Thromb Vasc Biol $2003 \cdot 23: 85-9$

32. Ryan AS, Berman DM, Nicklas BJ, Sinha M, Gingerich R, Meneilly GS, et al. Plasma adiponectin and leptin levels, body composition and glucose utilisation in adult women with wide ranges of obesity. Diabetes Care 2003;26:2383-88.

33. Kadowaki T, Yamauchi T, Kubota N, Hara K, Ueki K, Tobe K. Adiponectin and adiponectin receptors in insulin resistance, diabetes, and the metabolic syndrome. J Clin Invest 2006;116:1784-92.

34. Pischon T, Girman CJ, Hotamisligil GS, Rifai N, Hu FB, Rimm EB Plasma adiponectin levels and risk of myocardial infarction in man. JAMA. 2004;291:1730-37.

35. Ouchi N, Shibata R, Walsh K. Cardioprotection by adiponectin. Trends Cardiovasc Med. 2006; 16:141-46.

36. Taborsky GJ, Havel PJ, Porte D. Stres-induced activation of the neuroendocrine system and its effects on carbohydrate metabolism. In: Ellenberg \& Rifkin's Diabetes Mellitus 1997; Porte D, Sherwin RS (eds). Appletion \& Lange 5th ed. USA, 141-51.

37. Brochu M, Poehlman ET, Ades PA. Obesity, body fat distribotion, and corornaty artey disease. J Cardiopulmonary Rehabil 2000; 20: 96-108.

38. Alberti KGMM, Zimmet P, Shaw J. Metabolic Syndrome- a new worldwide definition. A concensus statement from the International Diabetes Federation. Diabetic Medicine. 2006;23:469-80.

39. Özenoğlu A., Can G, Hatemi H. Body composition reference values of adult women and men grouped as normal weight, overweight, obese and morbidly obese according to the body mass index.

40. Grundy SM, Hansen B, Smith SC, Cleeman CI, Kahn RA. Clinical treatment of the metabolic syndrome. AHA/NHLBI/ADA conrefence report. Circulation. 2004;109:551-56.

41. Third Report of the National Cholesterol Education Program (NCEP) expert panel on detection, evaluation, and treatment high blood cholesterol in adults (Adult Treatment Panel III): final report. Circulation. 2002;106:3143-3421.

42. Amamoto T, Kumai T, Nakaya S, Matsumoto N, Tsuziki Y, Kobayashi $\mathrm{S}$. The elucidation of the mechanism of wei ht gain and glucose tolerance abnormalities induced by chlorpromazine. J Pharmacol Sci. 2006;102:213-19.

43. Ferretti G, Baccetti T, Moroni C, Savino S, Liuzzi A, Balzola F, Bicchiega V. Paraoxanase

activity in high density lipoproteins: Comparision between healthy and obese females. J Clin Endocrinol Metab. 2005;90:1728-33.

44. Cellini E, Tedde A, Bagnoli S, Nacmias B, Piacentini S, Bessi V, Baracco L, Sorbi S. Association analysis of the paraoxanase-1 gene with Alzheimer's disease. Neuroscience Letters. 2006;408:199-202.

45. Dantoine TF, Debord J, Merle L, Lacroix-Ramiandrisoa H, Bourzeix L, Charmes JP. Paraoxanase I activity: A new vascular marker of dementia? Ann N Y Acad Sci 2002; 977: 96-101. 
46. Jaouad L, Milochevitch C, Khahil A. PON 1 paraoxanase activity is reduced during HDL oxidation and is an indicator of HDL antioxidant capacity. Free Radical Res. 2003; 37:77-83.

47. Ferretti G, Bacchetti C, Marotti E, Carutola G. Effect of homocysteinylation on human high-density lipoproteins: a correlation with paraoxanase activity. Metabolism. 2003;52:146-51.

48. Yamada A, Shoji T, Tahara H, Emoto M, Nishizawa Y. Effect of insulin resistance on serum paraoxanase activity in a nondiabetic population. Metab. 2001;50:805-11.
49. Rosmond R, Björntorp P. The hypothalamic-pituitary-adrenal axis activity as a predictor of cardiovascular disease, type 2 diabetes and stroke. J Intern Med. 1999.

50. Groop L, Orho-Melander M. The dysmetabolic syndrom. Journal of Internal Medicine. 2001; 250:105-20. 